University of Nebraska - Lincoln

DigitalCommons@University of Nebraska - Lincoln

Faculty Papers and Publications in Animal

Science

Animal Science Department

2018

\title{
The impact of truncating data on the predictive ability for single- step genomic best linear unbiased prediction
}

\author{
Jeremy T. Howard \\ University of Nebraska-Lincoln, s-jhowar20@unl.edu \\ Thomas A. Rathje \\ University of Nebraska-Lincoln, trathje2@unl.edu \\ Caitlyn E. Bruns \\ DNA Genetics, Columbus, Nebraska \\ Danielle F. Wilson-Wells \\ DNA Genetics, Columbus, Nebraska, twinonedil@gmail.com \\ Stephen D. Kachman \\ University of Nebraska-Lincoln, steve.kachman@unl.edu \\ See next page for additional authors
}

Follow this and additional works at: https://digitalcommons.unl.edu/animalscifacpub

Part of the Applied Statistics Commons, Biostatistics Commons, Design of Experiments and Sample Surveys Commons, Genetics and Genomics Commons, Meat Science Commons, Other Mathematics Commons, Statistical Models Commons, and the Vital and Health Statistics Commons

Howard, Jeremy T.; Rathje, Thomas A.; Bruns, Caitlyn E.; Wilson-Wells, Danielle F.; Kachman, Stephen D.; and Spangler, Matthew L., "The impact of truncating data on the predictive ability for single-step genomic best linear unbiased prediction" (2018). Faculty Papers and Publications in Animal Science. 1018.

https://digitalcommons.unl.edu/animalscifacpub/1018

This Article is brought to you for free and open access by the Animal Science Department at DigitalCommons@University of Nebraska - Lincoln. It has been accepted for inclusion in Faculty Papers and Publications in Animal Science by an authorized administrator of DigitalCommons@University of Nebraska - Lincoln. 


\section{Authors}

Jeremy T. Howard, Thomas A. Rathje, Caitlyn E. Bruns, Danielle F. Wilson-Wells, Stephen D. Kachman, and Matthew L. Spangler 


\title{
The impact of truncating data on the predictive ability for single-step genomic best linear unbiased prediction
}

\author{
Jeremy T. Howard, ${ }^{1}$ Tom A. Rathje, ${ }^{2}$ Caitlyn E. Bruns, ${ }^{2}$ \\ Danielle F. Wilson-Wells, ${ }^{2}$ Stephen D. Kachman, ${ }^{3}$ \\ and Matthew L. Spangler ${ }^{1}$
}

\author{
1 Department of Animal Science, University of Nebraska-Lincoln, Lincoln, Nebraska \\ 2 DNA Genetics, Columbus, Nebraska \\ 3 Department of Statistics, University of Nebraska-Lincoln, Lincoln, Nebraska \\ Corresponding author - Jeremy T. Howard, Department of Animal Science, University of Nebraska- \\ Lincoln, Lincoln, NE 68583-0908; email s-jhowar20@unl.edu
}

ORCID: Jeremy T. Howard 0000-0001-6216-3540

\begin{abstract}
Simulated and swine industry data sets were utilized to assess the impact of removing older data on the predictive ability of selection candidate estimated breeding values (EBV) when using single-step genomic best linear unbiased prediction (ssGBLUP). Simulated data included thirty replicates designed to mimic the structure of swine data sets. For the simulated data, varying amounts of data were truncated based on the number of ancestral generations back from the selection candidates. The swine data sets consisted of phenotypic and genotypic records for three traits across two breeds on animals born from 2003 to 2017. Phenotypes and genotypes were iteratively removed 1 year at a time based on the year an animal was born. For the swine data sets, correlations between corrected phenotypes (Cp) and EBV were used to evaluate the predictive ability on young animals born in 2016-2017. In the simulated data set, keeping data two generations back or greater resulted in no statistical difference $(p$-value $>0.05)$ in the reduction in the true breeding value at generation 15 compared to utilizing all available data. Across swine data sets, removing phenotypes from animals born prior to 2011 resulted in a negligible or a slight numerical increase in the correlation between Cp and EBV. Truncating data is a method to alleviate computational issues without negatively impacting the predictive ability of selection candidate EBV.
\end{abstract}

Keywords: data reduction, single-step genomic BLUP, swine 


\section{Introduction}

The use of genomic information to predict breeding values, referred to as "genomic selection" (Meuwissen, Hayes, \& Goddard, 2001), has revolutionized animal breeding across the majority of livestock species (Knol, Nielsen, \& Knap, 2016). The premise behind genomic selection is centered around the concept of generating a training population containing animals with genomic and phenotypic information. The training population is utilized to estimate the effects of genome-wide markers across multiple traits. The associated marker effects are then used to predict genomic estimated breeding values (GEBV) for genotyped selection candidates. Based on this premise, research has been conducted to understand what factors impact the erosion of GEBV accuracy when phenotypes are not collected routinely (Bastiaansen, Coster, Calus, van Arendonk, \& Bovenhuis, 2012; Long, Gianola, Rosa, \& Weigel, 2011).

At present, the landscape of genomic selection is changing such that animals are being routinely genotyped along with, in general for most traits, phenotypes being routinely collected. Moreover, if marker effects are assumed normally distributed with a constant variance, genomic selection as outlined previously is equivalent to replacing the pedigree-based relationship matrix $(\mathbf{A})$ with a genomic-based relationship matrix $(\mathbf{G})$ in the best linear unbiased prediction (BLUP) equations (Hayes, Bowman, Chamberlain, Verbyla, \& Goddard, 2009). Furthermore, a method that augments pedigree relationships across all individuals based on contributions from animals with genomic information was developed and is referred to as single-step genomic BLUP (ssGBLUP; Aguilar et al., 2010; Christensen \& Lund, 2010). As a result, phenotype, genotype and pedigree information on all animals are utilized simultaneously (i.e., no training and validation population) in the BLUP equations.

The pace at which genotypes accumulate within a species continues to increase across the majority of livestock species. For example, the number of genotyped Holstein animals in the United States exceeds 1.5 million (https://queries.uscdcb.com/Genotype/counts.html). As outlined in Misztal, Legarra, and Aguilar (2014), as the number of genotyped animals gets large (i.e., greater than 100,000), computational issues arise, and therefore, alternative representations of $\mathbf{G}$ have been proposed (Misztal et al., 2014). By instinct, as generational information accrues in a breeding population, selection candidates become more distantly related to the majority of the historic population, thus eroding the benefit of data (i.e., phenotype, pedigree and genotype information) from historic animals relative to predicting the genetic merit of the most recent generation of selection candidates. 
Furthermore, trait definitions as a result of improved phenotype collection technology, change in the genetic architecture and/or models may change across time. These changes coupled with potential parentage errors create a situation where information on older animals potentially negatively impacts the accuracy of selection candidates. Therefore, the objective of this study was to assess the impact of removing older data in the form of phenotype, genotype and pedigree information utilizing simulation and empirical swine data sets.

\section{Materials and methods}

No animal care approval was required because all records came from simulated data or an existing industry database.

\subsection{Simulated data}

To investigate the long-term impacts of continually removing data across generations on the genetic gain and the associated computational reductions, a simulation was conducted using the Geno-Diver software (Howard, Tiezzi, Pryce, \& Maltecca, 2017; V2). The simulation was generated to closely resemble the population structure of a swine nucleus population. The genome was assumed to consist of five chromosomes that were each $136 \mathrm{Mb}$ long. The value of 136 was chosen based on the mean length of a swine autosomal chromosome. The simulation was initialized by calling the coalescence-based simulation program MaCS (Chen, Marjoram, \& Wall, 2009) within Geno-Diver to generate SNP sequence data for 1,800 base haplotypes for each chromosome (Chen et al., 2009). To generate moderate short-range linkage disequilibrium that is similar to livestock species, the "Ne100_Scen1" option within Geno-Diver was utilized to generate sequence data for the founder population. The "Ne100_Scen1" option sets the effective population size in the founder population to 100 . Once the base haplotypes were generated, 200 randomly placed quantitative trait loci (QTL) were generated within each chromosome, which resulted in a total of 1,000 QTL across all chromosomes. A marker panel consisting of 15,000 (i.e., 3,000 markers per chromosome) neutral markers was constructed. The number of markers per chromosome was chosen to resemble a medium density marker panel (i.e., Illumina PorcineSNP60K BeadChip; IIlumina Inc.). In order for a QTL or marker to be chosen from the full set of base haplotypes, the minor allele frequency (MAF) had to be greater than 0.01 and 0.05 , respectively. 
The additive effects $(a)$ for a QTL were generated using a gamma distribution (shape $=0.4$ ), with an equal chance of being positive or negative. The phenotype for an individual $\left(\mathrm{y}_{i}\right)$ was generated as:

$$
y_{i}=\mu+\sum_{q=1}^{n Q T L} \gamma_{i q} a_{q}+e_{i}
$$

where $\mu$ is the general mean, $n Q T L$ is the number of QTL, $\gamma_{i q}$ is the genotype (i.e., 0 for the homozygote; 1 for the heterozygote; 2 for the alternative homozygote) for individual $i$ at QTL $q, a_{q}$ is the additive effect for QTL $q$, and $e$ is a random residual $\left(e \sim \mathrm{N}\left(0, \sigma_{e}^{2}\right)\right.$ ) for individual $i$. The additive effects were scaled to generate a trait with a heritability of 0.10 . The phenotypic variance was set at 1.0; therefore, the residual variance was 0.90 . At first, both a low and moderate $\left(h^{2}=0.35\right)$ heritability scenario were investigated and no differences were observed. As a result, only the low heritability scenario will be described.

The founder population consisted of 50 males and 500 females that were generated by randomly allocating base haplotypes, without replacement, to founder individuals across all chromosomes. After the founder population was initialized, individuals were generated using a forward-in-time simulation approach for 15 generations. The population size for the forward-intime simulation was 50 males and 500 females, with a replacement rate of 0.60 across both sexes. An animal was allowed to remain in the breeding population for a maximum of eight generations. All parents were mated at random, and the number of matings was the same across all sires (i.e., 10 matings per sire) and each mating resulted in six offspring that were available as selection candidates for the next generation. To build up the pedigree, four generations of random selection and culling were utilized. For the remaining generations, animals were selected and culled based on their EBV. Within a generation, a maximum of two selection candidates could be selected within each full-sib family.

Two phenotyping and genotyping strategies were investigated to determine the impact of data truncation with different amounts of information available on the selection candidates when EBV were estimated. In the scenario with minimal information on the selection candidates (Scenario 1), it was assumed that an animal obtained its own phenotypic information on a trait after the selection step and only progeny selected as parents in the next generation were genotyped (i.e., no genotype information on selection candidates). Due to selection candidates lacking phenotypic and genotypic information, all full-sib selection candidate EBV were based on the average EBV of their parents. Therefore, selection candidates within a full-sib family were randomly selected. In the scenario with more information on the selection candidates (Scenario 2), it was assumed that selection candidates had 
phenotype and genotype information at the time of selection and all parents had genotypic information. Under this scenario, full-sib selection candidate EBV are not all equal, as was the case in Scenario 1.

The EBV were estimated based on an animal model as outlined below:

$$
y=\mathbf{X b}+\mathbf{Z u}+\mathrm{e}
$$

Model 1

where $y$ is a vector of phenotypic observations, $b$ is a vector of fixed effects, $u$ is a vector of random additive genetic effects, e is a vector of random residuals, and $\mathbf{X}$ and $\mathbf{Z}$ are incidence matrices relating observations to the fixed and random additive genetic effects, respectively. The only fixed effect was the intercept. The additive genetic and environmental variance was assumed to be 0.10 and 0.90 , respectively, and remained constant across generation. Breeding values were estimated using the preconditioned conjugate gradient algorithm (Lidauer, Strandén, Mäntysaari, Pösö, \& Kettunen, 1999). In both scenarios, the potential for an animal to be genotyped started at generation 8 and continued for all remaining generations. Across both scenarios, all parents in the breeding population at generation 8 that were selected to serve as parents in a previous generation and have not been culled were genotyped. As a result, EBV from generations 5-8 were estimated based on a traditional pedigree relationship matrix $\left(\mathbf{A} ; u \sim N\left(0, \sigma_{u}^{2} \mathbf{A}\right)\right)$. Pedigree-based inbreeding values were estimated based on the method outlined in Meuwissen and Luo (1992) and utilized when generating the inverse of $\mathbf{A}$ based on Henderson (1976). Starting at generation 9, breeding values were estimated based on ssGBLUP, which blends pedigree and genomic information, referred to as $\mathbf{H}\left(u \sim N\left(0, \sigma_{u}^{2} \mathbf{H}\right)\right.$ ) (Aguilar et al., 2010; Christensen \& Lund, 2010). An initial $G$ matrix $\left(\mathbf{G}_{\text {raw }}\right)$ was constructed as

$$
\mathbf{G}_{\text {raw }}=\frac{\mathbf{M M}^{\prime}}{2 \sum p_{j}\left(1-p_{j}\right)}
$$

where $\mathbf{M}$ is a genotype incidence matrix that has been centered based on allele frequencies (VanRaden, 2008), and $p$ is the allele frequency of the second allele at the $j$ th SNP. The allele frequencies were estimated from all genotyped animals that were utilized when estimating breeding values. Therefore, as generations accrue, the allele frequencies used to create $G_{\text {raw }}$ change as new animals get genotyped and/or older genotyped animals get truncated. As outlined in Vitezica, Aguilar, Misztal, and Legarra (2011), $\mathbf{A}_{\mathbf{2 2}}$ and $\mathbf{G}_{\text {raw }}$ need to be compatible. The $\mathbf{A}_{\mathbf{2 2}}$ matrix refers to the pedigree-based relationship for genotyped animals and was constructed as outlined in Colleau (2002). Therefore, $\mathbf{G}_{\text {raw }}$ was adjusted to make the mean diagonal and mean of all elements equal the mean diagonals and mean of all elements of $\mathbf{A}_{22}$ as outlined in Christensen, Madsen, Nielsen, Ostersen, and Su (2012). Last 
of all, prior to blending $\mathbf{G}$ with pedigree information, a weighted $\mathbf{G}$ as proposed by VanRaden (2008) was constructed as $\mathbf{G}=0.95 \mathbf{G}_{\text {raw }}+0.05 \mathbf{A}_{\mathbf{2 2}}$ and the associated matrix was utilized when constructing $\mathbf{H}$.

To understand the impact of removing data from older individuals, phenotypic, pedigree and genomic data were progressively removed when predicting the genetic merit of the current generation of selection candidates. The data truncation points were 1, 2, 3, 4, 5 and 10 ancestral generations back from the selection candidates. Within each generation, data from relatives that remained after truncation and their associated offspring were used to generate EBV within each of the truncation point scenarios listed above. The impact of a given truncation point was compared to utilizing all available data when predicting the genetic merit of selection candidates. Thirty replicates were generated within each data truncation point. The genetic trend across truncation points was determined along with the correlation between the true breeding value (TBV) and EBV on the selection candidates within each generation. Last of all, the bias of EBV across different truncation points within each generation for the selection candidates was quantified by the coefficient of regression of TBV on EBV. The ideal coefficient of regression is a value of 1.0, which implies the EBV are unbiased. For each metric, the $95 \%$ confidence interval was calculated across all replicates based on a randomized complete block design with replicates (i.e., block) and truncation methods considered fixed.

\subsection{Empirical data}

\subsubsection{Animals and genotypes}

The empirical data sets were utilized to verify results from simulation based on the predictive ability of selection candidate EBV for animals born recently when data from older animals were progressively removed. Data from a maternal (Yorkshire) and terminal (Duroc) purebred nucleus selection line were obtained from DNA Genetics (Columbus, NE). Within each population, the following traits were investigated: ultrasonic backfat (BF), ultrasonic loin eye depth (LED) and average daily gain in the nursery (ADGn). For Yorkshire, the data consisted of phenotyped animals (ADGn: $n=117,875$; BF and LED: $n$ $=95,769$ ) born from 2005 to 2017, along with 5,783 genotyped animals. Of the genotyped animals, $31.4 \%$ were males and $68.6 \%$ were females. For Duroc, the data consisted of phenotyped animals (ADGn: 152,302; BF and LED: 135,327) born from 2003 to 2017 along with 12,180 animals with genotypes. Of the genotyped animals, $39.2 \%$ were males and $60.8 \%$ were females. In both populations, genotyped animals were born from 2011 to 2017 . The 


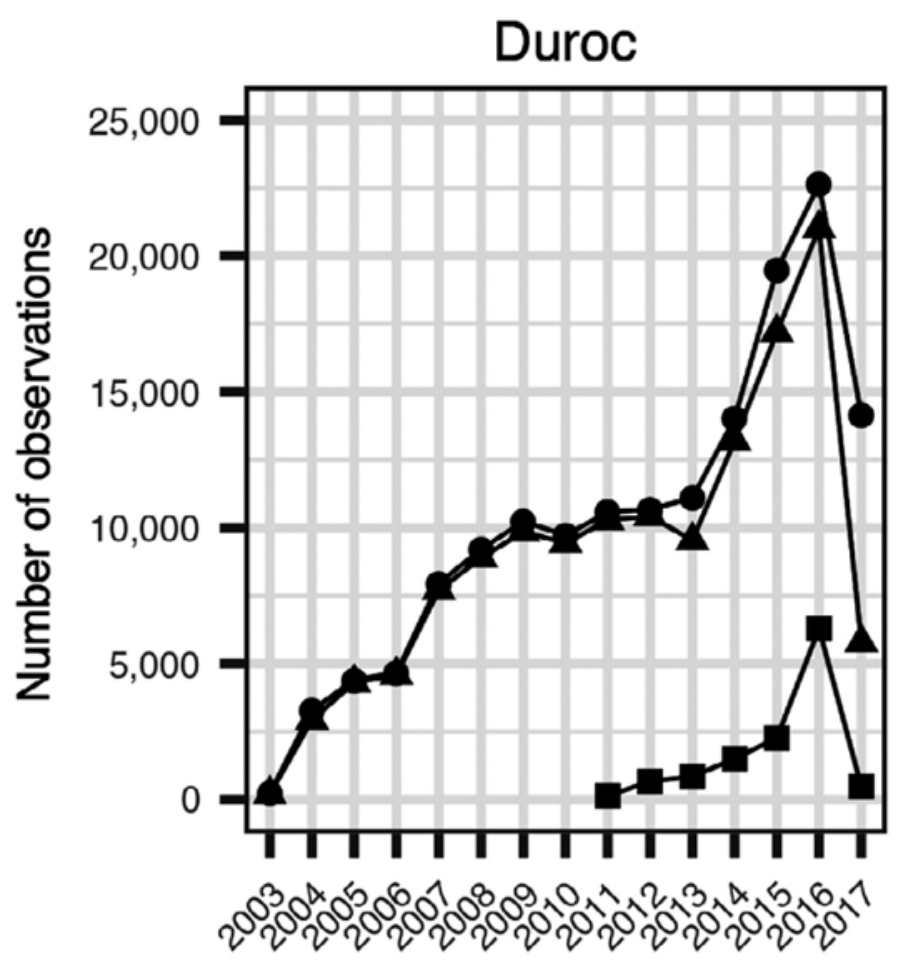

Birth year

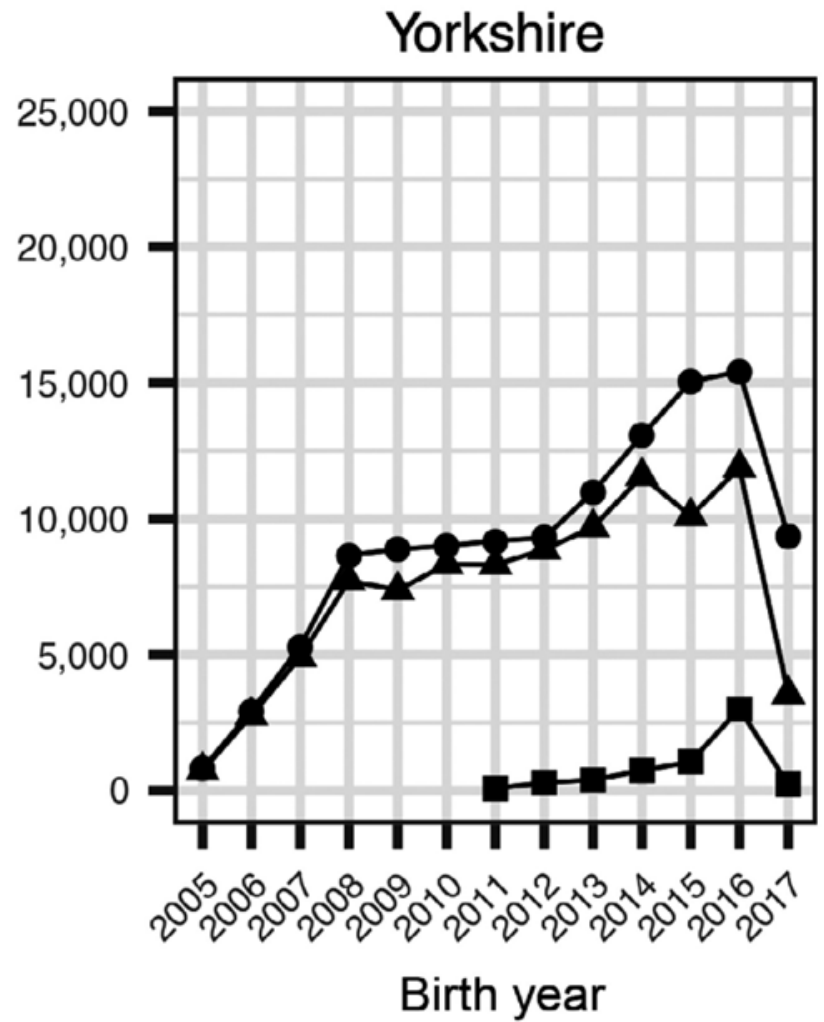

BF \& LED

Genotypes

Figure 1. Number of phenotypic observations by trait ${ }^{1}$ and genotypes by year of birth for the Duroc and Yorkshire populations.

1. ADGn refers to average daily gain in the nursery; BF refers to backfat; LED refers to loin eye depth.

number of animals with phenotypic and genomic information by year of birth is outlined in Figure 1. In both populations, genotypes were derived from a Affymetrix SNP array (Affymetrix, Inc., Santa Barbara, CA) that is comprised of 55,774 SNP spread uniformly across the genome. Prior to the imputation of missing genotypes, multiple quality control edits were conducted including removing SNP with missing map information and those on the sex chromosome, the removal of individuals and SNPs with call rates lower than 0.90, SNP with a MAF lower than 0.002 and a $p$-value of a chi-squared test for Hardy-Weinberg equilibrium lower than 0.0001. Imputation of missing genotypes was conducted using Flmpute (Sargolzaei, Chesnais, \& Schenkel, 2014). After quality control and imputation of missing genotypes, a total of 41,279 and 34,796 SNP remained for Yorkshire and Duroc, respectively. 


\subsubsection{Model and truncation description}

Within each trait, a single-trait ssGBLUP animal model was utilized within the blupf90 suite of programs (Misztal et al., 2002). Similar to the analysis of simulated data, $\mathbf{H}$ was constructed utilizing a weighted $\mathbf{G}$ (i.e., $\mathbf{G}=0.95 \mathbf{G}_{\text {raw }}$ $+0.05 \mathbf{A}_{\mathbf{2 2}}$ ) and adjusted to make $\mathbf{G}_{\text {raw }}$ and $\mathbf{A}_{\mathbf{2 2}}$ compatible. The model for ADGn, BF and LED was:

$$
\begin{aligned}
& \mathbf{y}=\mathbf{X b}+\mathbf{L} 1+\mathbf{Z u}+\mathrm{e}_{\mathrm{r}} \quad \text { ADGn; Model } 2 \\
& \mathbf{y}=\mathbf{X b}+\mathbf{L} 1+\mathbf{P p}+\mathbf{Z u}+\mathrm{e}, \quad \text { BF and LED; Model } 3
\end{aligned}
$$

where $y$ is a vector of phenotypic observations, $b$ is a vector of fixed effects, $I$ is a vector of random common litter environmental effects, $p$ is a vector of random pen effects, $u$ is a vector of random additive genetic effects, $\mathrm{e}$ is a vector of random residuals, and $\mathbf{X}, \mathbf{L}, \mathbf{P}$ and $\mathbf{Z}$ are incidence matrices relating observations to the fixed, random common litter environmental, random pen and random additive genetic effects, respectively. The fixed effects for ADGn included the intercept, sex, barn, contemporary group (i.e., yearbarn-month of birth) and age on test deviated from the mean (77 days) as a covariate. The fixed effects for BF and LED included the intercept, sex, contemporary group (i.e., year-barn-group) and off-test weight as a covariate. Across all traits and populations, the pedigree was truncated to three ancestral generations back from animals with phenotypic information. Variance components within each population and trait were estimated utilizing all available information and were fixed across different data truncation points.

The impact of removing older data was determined by masking the phenotype when predicting the EBV for animals born recently and served as the validation population. The validation population was comprised of genotyped and nongenotyped animals. Across both populations, animals born after December 2016 served as the validation population. The validation population for BF and LED consisted of 5,681 (genotyped = 776; nongenotyped $=4,905$ ) and 9,382 (genotyped $=1,371$; nongenotyped $=8,011$ ) animals for Yorkshire and Duroc, respectively. The validation population for ADGn consisted of 12,122 (genotyped $=776$; nongenotyped $=11,346$ ) and 17,932 (genotyped $=1,372$; nongenotyped $=16,559$ ) animals for Yorkshire and Duroc, respectively. The phenotypes and genotypes (when available) were iteratively removed 1 year at a time starting with the oldest year and ending with 2015. Therefore, data were truncated based on the year an animal was born, not by the ancestral generation number, which was done in the simulation. Truncation based on year was chosen due to the varying amounts of phenotypic and genotypic information across the traits investigated. As the TBV is not known in the empirical data, the correlation between the EBV 
within a given truncation scenario and the corrected phenotype (Cp) was utilized to determine the loss/gain in the predictive ability when older data were removed. The $\mathrm{C} p$ was estimated by adjusting for all fixed and random effects except for the additive genetic and residual effects. The correlation between EBV and Cp results is presented by combining both genotyped and nongenotyped individuals. The nongenotyped and genotyped individuals were combined because the maximum correlation between $\mathrm{Cp}$ and EBV within the nongenotyped and genotyped subgroup is in general similar across the majority of the traits within each breed.

\section{Results}

\subsection{Simulated data}

The mean TBV of the selection candidates at generation 15 across different truncation points and genotyping scenarios is illustrated in Table 1. Keeping data only one generation back from the selection candidates resulted in a $10.5 \%$ and $6.0 \%$ reduction in the TBV at generation 15 compared to utilizing all available data, for Scenario 1 and Scenario 2, respectively. Keeping data two generations back or greater resulted in no statistical difference ( $p$ value $>0.05$ ) in the reduction in TBV at generation 15 compared to utilizing

Table 1. Mean ( $\pm 95 \%$ confidence interval) true breeding value (TBV) for the selection candidates at generation 15 for a given truncation point (i.e., ancestral generations).

\begin{tabular}{lll} 
& \multicolumn{2}{l}{ Simulation scenario $^{b}$} \\
\cline { 2 - 3 } Truncation point & & \\
\hline Full & Scenario 1 & Scenario 2 \\
10 & $1.579(1.543-1.615)$ & $2.635(2.602-2.668)$ \\
5 & $1.643(1.607-1.680)$ & $2.644(2.611-2.677)$ \\
4 & $1.625(1.588-1.661)$ & $2.623(2.590-2.656)$ \\
3 & $1.611(1.574-1.647)$ & $2.626(2.594-2.659)$ \\
2 & $1.606(1.569-1.642)$ & $2.602(2.570-2.635)$ \\
1 & $1.560(1.524-1.597)$ & $2.621(2.588-2.654)$ \\
\hline
\end{tabular}

a. Truncation point refers to utilizing information on relatives and their associated progeny that are 1, 2, 3, 4, 5, 10 ancestral generation generations back from the selection candidates when breeding values were estimated. Under the full truncation scenario, all available information was utilized when EBV were estimated within each generation.

b. Scenario 1 refers to selection candidates not having phenotype or genotype information when EBV are estimated; Scenario 2 refers to selection candidates having phenotype and genotype when EBV are estimated. 

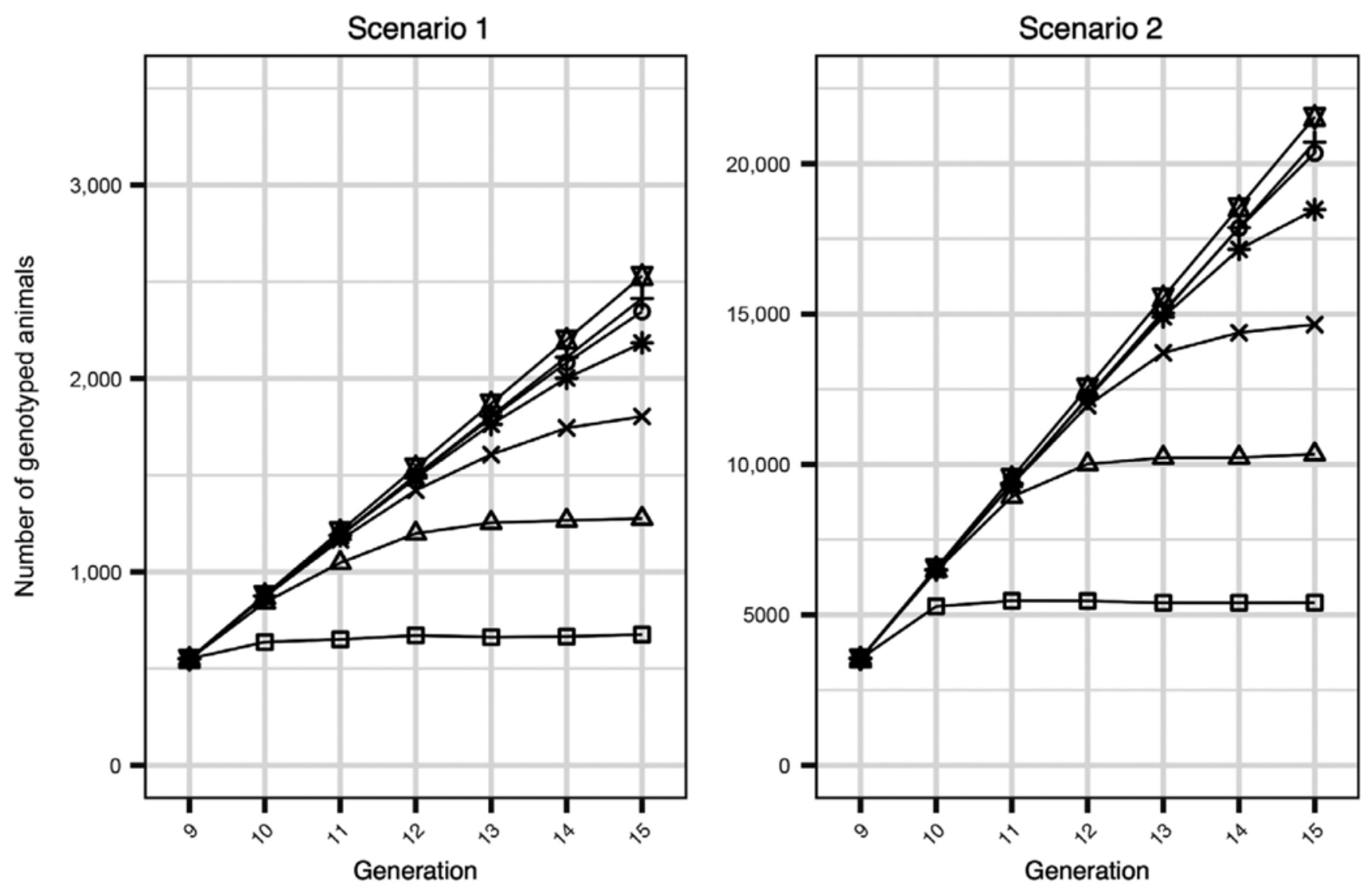

Truncation point
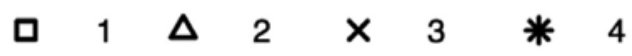

$05+10$ full

Figure 2. Number of genotyped animals utilized in the prediction of breeding values across different truncation points ${ }^{1}$ and genotyping scenarios ${ }^{2}$ in the simulated data.

1. Truncation point refers to utilizing information on relatives and their associated progeny that are 1,2 , $3,4,5,10$ ancestral generation generations back from the selection candidates when breeding values were estimated. Under the full truncation scenario, all available information was utilized when EBV were estimated within each generation.

2. Scenario 1: Selection candidates did not have phenotype and genotype information when estimated breeding values (EBV) were predicted. Scenario 2: Selection candidates had phenotype and genotype information when EBV were predicted.

all available data. Across both genotyping scenarios, the numerically largest TBV at generation 15 occurred when data were truncated at 10 generations although the differences between truncations points after two generations back were not statistically different ( $p$-value $>0.05)$. The reduction in the number of genotyped animals utilized when constructing $\mathrm{H}$ across different truncation points across both genotyping strategies is illustrated 

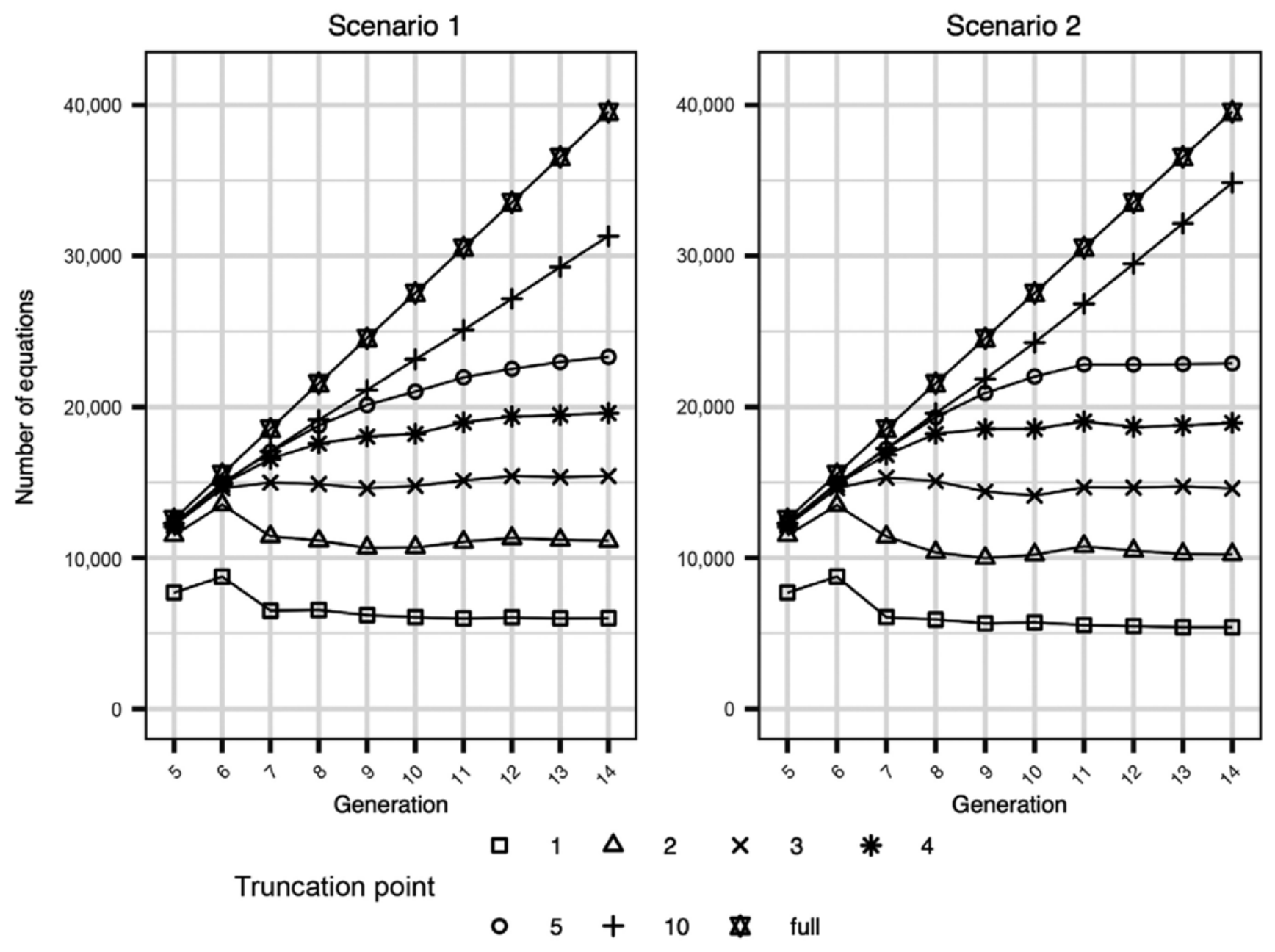

Figure 3. Number of equations to solve in the prediction of breeding values across different truncation points ${ }^{1}$ and genotyping strategies ${ }^{2}$ in the simulated data.

1. Truncation point refers to utilizing information on relatives and their associated progeny that are 1,2 , $3,4,5,10$ ancestral generation generations back from the selection candidates when breeding values were estimated. Under the full truncation scenario, all available information was utilized when EBV were estimated within each generation.

2. Scenario 1: Selection candidates did not have phenotype and genotype information when estimated breeding values (EBV) were predicted. Scenario 2: Selection candidates had phenotype and genotype information when EBV were predicted.

in Figure 2. In the same way, the reduction in the number of equations to solve in the ssGBLUP model across different truncations points and genotyping strategies is outlined in Figure 3. Removing data in the form of phenotypes, genotypes and pedigree information resulted in a large reduction in the total number of equations to solve and reduced the computational burden when constructing $\mathbf{H}$, without negatively impacting the genetic trend. 

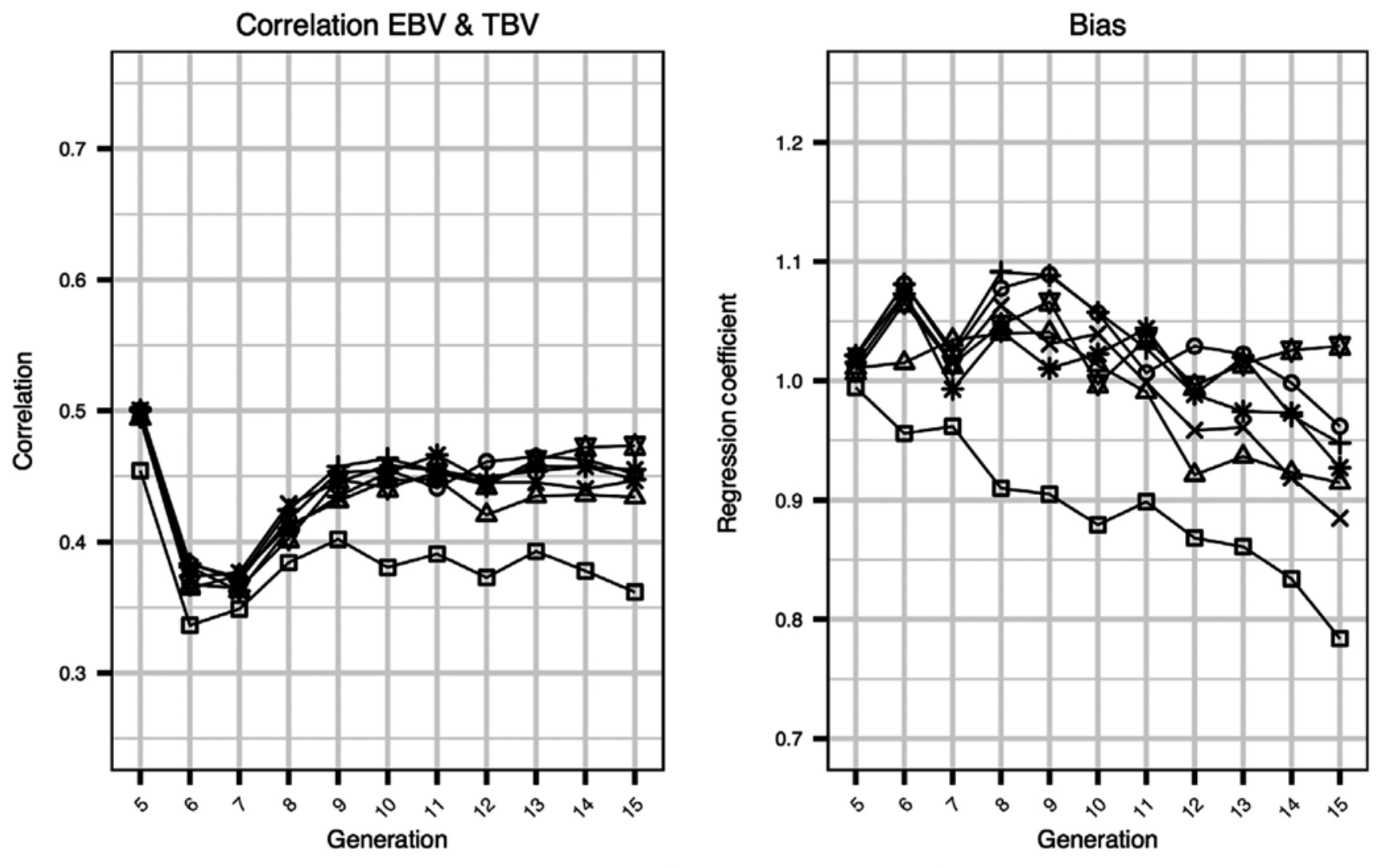

Truncation Point

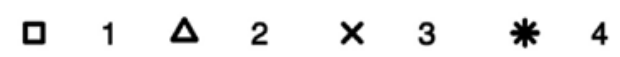

$05+10 \&$ full

Figure 4. Mean ${ }^{1}$ correlation between true breeding value (TBV) and estimated breeding value (EBV) and bias $^{2}$ across generations by truncation points ${ }^{3}$ for genotype Scenario $1^{4}$.

1. Mean $95 \%$ confidence interval surrounding point estimate was \pm 0.016 and 0.040 for the correlation between TBV and EBV and bias of EBV, respectively.

2. Bias was calculated as the regression of TBV on EBV.

3. Truncation point refers to utilizing information on relatives and their associated progeny that are 1,2 , $3,4,5,10$ ancestral generation generations back from the selection candidates when breeding values were estimated. Under the full truncation scenario, all available information was utilized when EBV were estimated within each generation.

4. Scenario 1 refers to selection candidates not having phenotype or genotype information when EBV are estimated.

For example, when truncating data at two generations back from the selection candidates for Scenario 1, the number of equations to solve at generation 14 was reduced by $72 \%$ and only $58 \%$ of the genotyped animals were utilized when constructing $\mathbf{H}$. A similar reduction in the number of equations to solve at generation 14 for Scenario 2 was observed (74\%), although the computational savings were larger compared to Scenario 1, due to the 

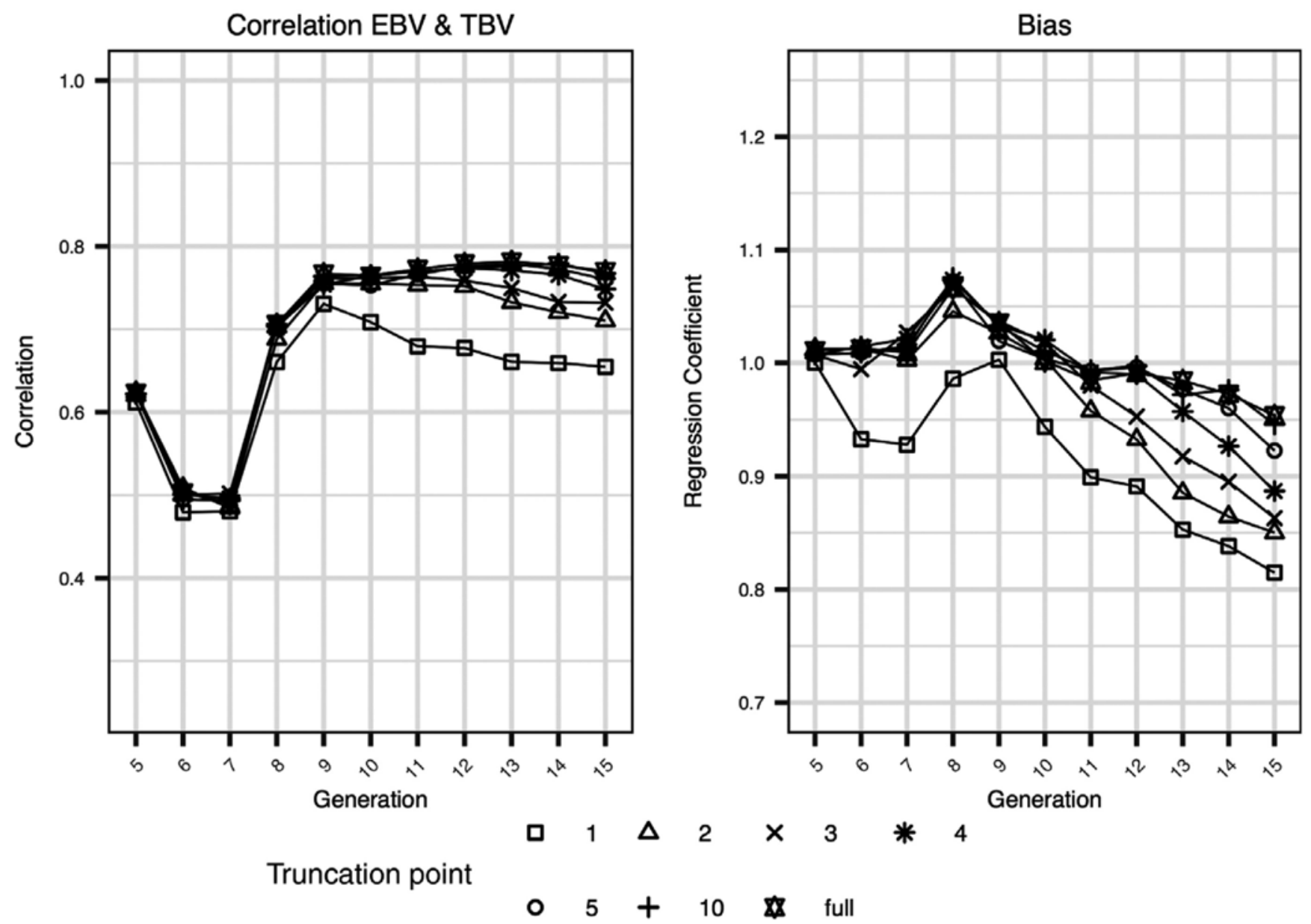

Figure 5. Mean ${ }^{1}$ correlation between true breeding value (TBV) and estimated breeding value (EBV) and bias $^{2}$ across generations by truncation points ${ }^{3}$ for genotype Scenario $2^{3}$.

1. Mean $95 \%$ confidence interval surrounding point estimate was \pm 0.009 and 0.020 for the correlation between TBV and EBV and bias of EBV, respectively.

2. Bias was calculated as the regression of TBV on EBV.

3. Truncation point refers to utilizing information on relatives and their associated progeny that are 1,2 , $3,4,5,10$ ancestral generation generations back from the selection candidates when breeding values were estimated. Under the full truncation scenario, all available information was utilized when EBV were estimated within each generation.

4. Scenario 2 refers to selection candidates having phenotype and genotype when EBV are estimated.

removal of over 8,000 genotyped animals when constructing $\mathbf{H}$. More important, as illustrated in Figure 2, across the latter generations, the number of genotyped animals utilized when constructing $\mathbf{H}$ began to stabilize across generations as new selection animals continued to be genotyped. Last of all, the correlation between the EBV and TBV along with the degree that EBV were inflated across generations is depicted in Figures $\mathbf{4}$ and $\mathbf{5}$ for Scenario 
1 and Scenario 2, respectively. Similar to the reduction in genetic gain results, a reduced correlation between EBV and TBV was observed when truncating the data, one generation removed from the selection candidates, across all generations for both genotyping scenarios. The initial generations display a greater degree of change as a result of initiating truncation selection along with a change in prediction method (i.e., going from pedigree-based BLUP to ssGBLUP). Across all truncation point cut-offs and both genotyping scenarios, the degree of bias was similar and in general was negligible, with values close to 1.0. However, as ssGBLUP selection proceeded, the bias coefficient deviated from one to a greater degree as the number of future generations increased, which is partially due to changes in the additive genetic variance as selection proceeds. This deviation was larger for Scenario 2, which had a larger selection response compared to Scenario 1.

\subsection{Empirical data}

The proportional increase/decrease in the correlation between $\mathrm{Cp}$ and the EBV across different truncation points based on the year an animal was born compared to the correlation based on utilizing all available data for Yorkshire and Duroc populations is depicted in Figure 6. Across both breeds and for all traits, the maximum correlation between $\mathrm{Cp}$ and EBV was observed when a proportion of the older data were removed. Across both populations, removing phenotypes from animals born prior to 2011 resulted in no decrease or a slight numerical increase in the correlation between C $p$ and EBV for selection candidates. Across all traits for Duroc, the largest correlation occurred when animals with phenotypes and genotypes born prior to 2013 were removed. For Duroc, the correlation (SE) for BF, LED and ADGn when utilizing all data was 0.1637 (0.0094), 0.1771 (0.0094) and 0.0750 (0.0072), respectively, compared to a correlation of 0.1650 (0.0094), $0.1786(0.0094)$ and 0.0772 (0.0072) when removing all data prior to 2013, respectively. For Yorkshire, the truncation point at which the largest correlation was estimated varied across traits. The correlation (SE) for LED and ADGn when utilizing all data was $0.1970(0.012)$ and $0.0743(0.0087)$, respectively, compared to $0.1985(0.012)$ and $0.0755(0.0083)$ and when removing all data prior to 2012, respectively. Last of all, the correlation for BF when utilizing all data was $0.1708(0.012)$, compared to $0.1710(0.012)$ when removing all data prior to 2011. Averaged across traits, when removing animals with phenotypes born prior to $2011,34 \%$ and $32 \%$ of the phenotypic data were not utilized for Duroc and Yorkshire, respectively. The year of birth for the genotyped animals was all relatively recent (i.e., all greater than 2011), and as a result, the impact of removing large cohorts of genotyped animals was not able to be fully investigated with the empirical data. However across both breeds, the numerically largest correlation was observed when a small portion of 


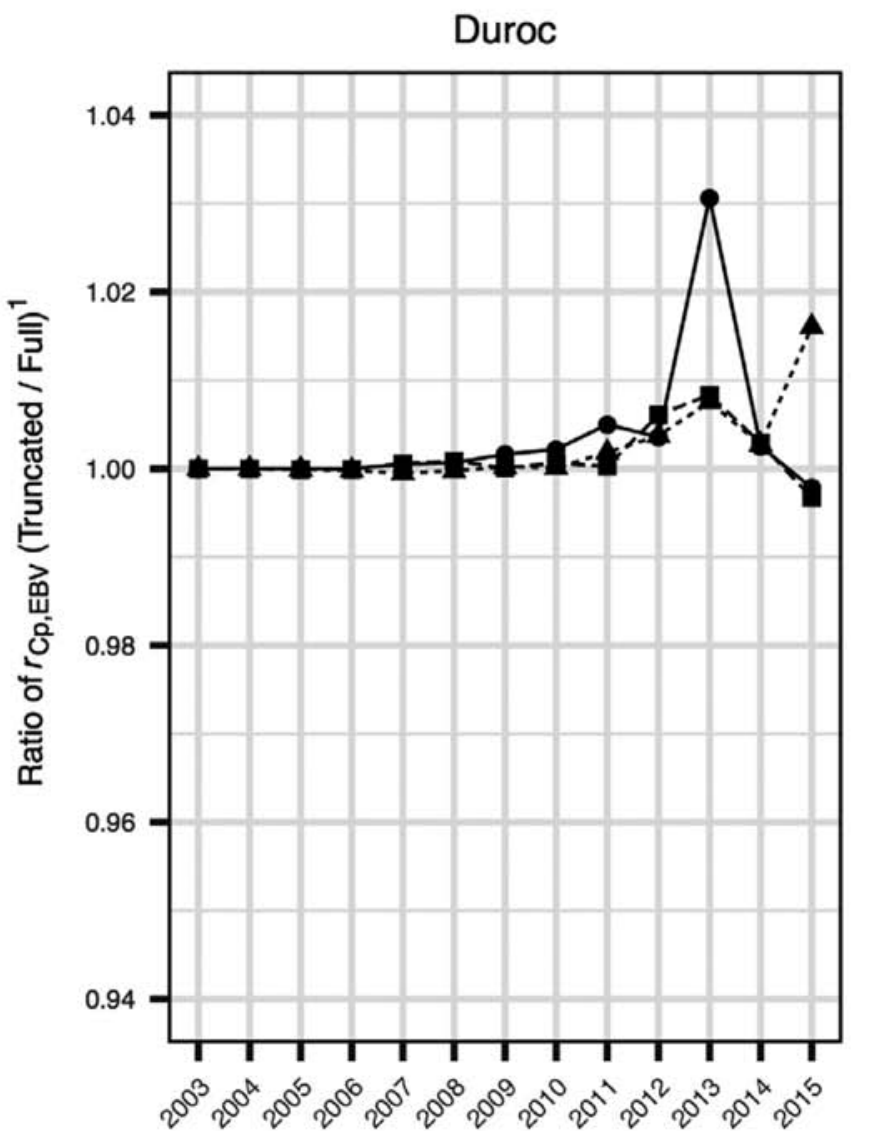

Birth year

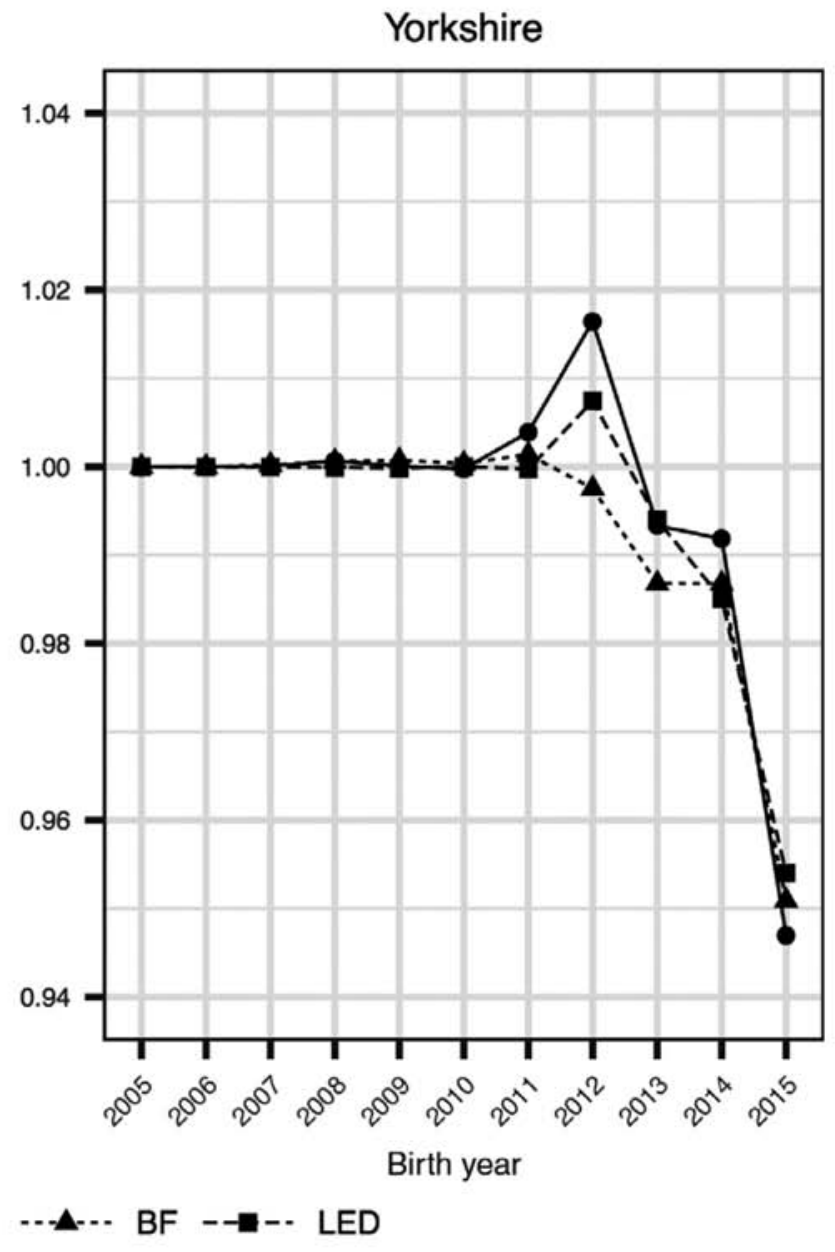

Figure 6. Ratio of the correlation ( $\left.r_{\mathrm{Cp}, \mathrm{EBV}}\right)$ between corrected phenotype $(\mathrm{Cp})$ and estimated breeding value (EBV) in the validation population for a given truncation point over $r_{\mathrm{Cp}, \text { EBV }}$ when utilizing all animals.

genotyped animals were removed, which provides preliminary evidence that removing genotype data in the empirical data set does not give rise to loss of information.

\section{Discussion}

The current study has investigated the impact of removing data, in the form of phenotype, genotype and pedigree information, from older animals based on simulated and empirical data. Due to the rapid uptake of genotyping animals across the majority of livestock species, a large amount of research 
has been conducted on methods to minimize the computational load and alleviate issues that arise when the number of genotyped animals becomes large (Fernando, Cheng, \& Garrick, 2016; Fragomeni et al., 2015; Misztal et al., 2014). The concept of truncating data in the form of pedigree information is not new within animal breeding, although the value of utilizing genotypes from older animals is currently not well understood. For example, Lush (1945, p 245-246) outlined that for practical purposes, pedigree relationships from animals four to five generations back from an animal are sufficient and any older ancestors are assumed to be a random sample of the breed. Furthermore, the expected proportion of an individual's genes from an ancestor is halved with each additional generation the ancestor is further back in the pedigree, although genomic information allows for variation from the expected proportion shared from a given ancestor. As a result, the impact of removing genotype information as generations accrue is potentially a viable option to alleviate the computational issues and reduce the computation time when estimating EBV without negatively impacting the selection candidate EBV accuracy.

A simulation was utilized to understand the impacts of removing data under an idealized condition, which includes no pedigree errors or genotyping errors, and the trait definition or breeding objective does not change across time. When keeping data two generations or greater back from the selection candidates, no statistical difference ( $p$-value $>0.05)$ in the mean TBV at generation 15 was observed compared to utilizing all available data across both genotyping strategies. When keeping data only one generation back (i.e., parents and associated progeny), the reduction in TBV was highly significant ( $p$-value $<0.0001)$ and reduced the TBV at generation 15 by $10.5 \%$ and $6.0 \%$ compared to utilizing all available data for Scenario 1 and Scenario 2 , respectively. The numerically largest mean TBV at generation 15 across both scenarios occurred when data were truncated and is partly due to $\mathbf{A}_{\mathbf{2 2}}$ and $\mathbf{G}$ being more compatible (i.e., larger diagonal and off-diagonal correlations). Averaged across generations, the correlation between the diagonal and off-diagonal values of $\mathbf{A}_{\mathbf{2 2}}$ and $\mathbf{G}$ across both genotyping scenario is outlined in Supporting information Table S1. If data truncation is practiced, the number of equations and the number of genotyped animals utilized become stationary at the latter generations of selection, despite continually collecting pedigree, phenotype and genotype information. Therefore, the set of genotyped animals utilized in genetic evaluation is updated with recently born selection candidates, while genotypes from older animals are no longer utilized. Across both genotyping scenarios, when data were truncated at two generations back from the selection candidates, the number of equations was reduced by 73 per cent at generation 14. Furthermore, 43 per cent of the genotypes were not utilized when estimating the breeding values of selection candidates at generation 14 . Under Scenario 1, only 
selection candidates selected to serve as parents were genotyped, and as illustrated in Figure 2, the total number of animals genotyped in the simulation was small compared to Scenario 2. Therefore, as a larger proportion of the animals born within a generation are genotyped, truncation of data results in even greater computational savings.

The correlation between the EBV and TBV was estimated across generations along with the bias of EBV. As expected based on the large reduction in TBV at generation 15, only keeping animals one generation back from the selection candidates resulted in a large reduction in the correlation between EBV and TBV across both genotyping scenarios. Furthermore, the bias across truncation methods had a 95\% confidence interval that contained 1.0 across the majority of generations, although some differences across truncation points did exist for the latter generations for both genotyping strategies. As outlined in Koivula, Strandén, Pösö, Aamand, and Mäntysaari (2015), different scaling methods for $\mathbf{G}$ and $\mathbf{A}_{\mathbf{2 2}}$ when setting up ssGBLUP will impact the degree that EBV are biased. The optimal scaling parameter or methods to construct the $\mathrm{H}$ matrix and its associated inverse is an active area of research (Garcia-Baccino et al., 2017) that will need to be further evaluated when data truncation techniques are utilized within a breeding program. Furthermore, the increase in bias in the latter generations is also partially due to the additive genetic variance changing as a result of truncation selection (Long et al., 2011) and older data being truncated when breeding values are being estimated.

Similar to the simulation results, across both the Yorkshire and Duroc populations the numerically largest EBV predictive ability occurred when data (phenotypes and genotypes) were truncated across all traits. Across both breeds, the maximum correlation between $\mathrm{Cp}$ and EBV occurred when removing 7 and 5 years of phenotypic data for Duroc and Yorkshire, respectively. For Duroc, the correlations ranged from 0.0750 to 0.1771 when utilizing all data and on average increased by 0.0017 when removing data prior to 2013 when the largest correlations were estimated. For Yorkshire, the correlations ranged from 0.0743 to 0.1985 when utilizing all data and on average increased by 0.001 when removing data prior to 2011 (BF) or 2012 (ADGn \& LED) when the largest correlations were estimated for these traits. Similar results were found by Lourenco et al. (2014), when phenotypic and pedigree data were progressively removed without any reduction in the accuracy of prediction EBV in a validation population, although the impact of removing genotypes was not evaluated. The impact of removing historical genotypes on the prediction accuracy of recently born selection candidates across multiple traits in chickens was investigated by Weng et al. (2016), and it was found that the optimal number of generations in the training varied from four or more for high heritability traits but less than that for low heritability traits. A slight numerical increase in the 
EBV predictive ability when information is removed is potentially due to a number of factors, which may include a change in trait definition across time or parentage errors in the pedigree. Nevertheless, when removing animals with phenotypes born prior to 2011, averaged across traits, 34\% and $32 \%$ of the phenotypic data were not utilized for Duroc and Yorkshire, respectively. Across both populations, the majority of animals with genotypes occurred within the past 3 years (i.e., 2014-2017), and as a result, the impact of removing large cohorts of genotyped animals was not able to be investigated. However, a small portion of the genotyped animals (6.5\%) for Duroc were removed when the maximum correlation between $\mathrm{Cp}$ and EBV was observed. For Yorkshire, no genotypes were removed when the maximum correlation between Cp and EBV was observed, although 1.4\% of the genotypes were removed when the maximum correlation between $C p$ and EBV was observed for ADGn and LED.

The empirical data utilized three traits that accrue a large amount of information across generations because they are recorded on a routine basis, can be recorded on both sexes and on live animals. Other traits such as sparsely recorded (e.g., individual feed intake), sex-limited reproductive traits, traits with a substantial maternal additive component or traits that occur later in life may result in different truncation points, and therefore, the truncation point is likely to be trait-specific. Furthermore, genetic evaluations are traditionally estimated based on multiple trait models, although singletrait models were utilized in the current analysis. The benefits of truncating data are expected to result in even greater computational savings although this needs to be researched further.

\section{Conclusion}

The current study has investigated the impact of removing older data, in the form of phenotypes, genotypes and pedigree information, on the prediction of EBV of selection candidates utilizing simulated and empirical data. Across both simulated and two swine industry data sets, removing data resulted in no change or a slight increase in the accuracy of predicting selection candidate EBV. Furthermore, the number of equations to solve and number of genotypes utilized when estimating breeding values can be greatly reduced. Truncating phenotypes, genotypes and pedigree information is a method to alleviate computational issues when the number of genotyped animals becomes large, without negatively impacting the prediction of selection candidate EBV.

\section{Additional supporting information follows the References.}




\section{References}

Aguilar, I., Misztal, I., Johnson, D. L., Legarra, A., Tsuruta, S., \& Lawlor, T. J. (2010). Hot topic: A unified approach to utilize phenotypic, full pedigree, and genomic information for genetic evaluation of Holstein final score. Journal of Dairy Science, 93, 743-752. doi 10.3168/jds.2009-2730

Bastiaansen, J. W. M., Coster, A., Calus, M. P. L., van Arendonk, J. A. M., \& Bovenhuis, H. (2012). Long-term response to genomic selection: effects of estimation method and reference population structure for different genetic architectures. Genetics Selection Evolution, 44, 3. doi 10.1186/1297-9686-44-3

Chen, G. K., Marjoram, P., \& Wall, J. D. (2009). Fast and flexible simulation of DNA sequence data. Genome Research, 19, 136-142. doi 10.1101/gr.083634

Christensen, O. F., \& Lund, M. S. (2010). Genomic predictions when some animals are not genotyped. Genetics Selection Evolution, 42, 2. doi 10.1186/1297-9686-42-2

Christensen, O. F., Madsen, P., Nielsen, B., Ostersen, T., \& Su, G. (2012). Singlestep methods for genomic evaluation in pigs. Animal, 6(10), 1565-1571. doi $10.1017 / \mathrm{S} 1751731112000742$

Colleau, J. J. (2002). An indirect approach to the extensive calculation of relationship coefficients. Genetics Selection Evolution, 34, 409-421. doi 10.1051/gse:2002015

Fernando, R. L., Cheng, H., \& Garrick, D. J. (2016). An efficient exact method to obtain GBLUP and single-step GBLUP when the genomic relationship matrix is singular. Genetics Selection Evolution, 48(1), 80. doi 10.1186/ s12711-016-0260-7

Fragomeni, B. O., Lourenco, D. A. L., Tsuruta, S., Masuda, Y., Aguilar, I., Legarra, A., ... Misztal, I. (2015). Hot topic: Use of genomic recursions in singlestep genomic best linear unbiased predictor (BLUP) with a large number of genotypes. Journal of Dairy Science, 98(6), 4090-4094. doi 10.3168/ jds.2014-9125

Garcia-Baccino, C. A., Legarra, A., Christensen, O. F., Misztal, I., Pocrnic, I., Vitezica, Z. G., \& Cantet, R. J. C. (2017). Metafounders are related to Fst fixation indices and reduce bias in single-step genomic evaluations. Genetics Selection Evolution, 49, 34. doi 10.1186/s12711-017-0309-2

Hayes, B. J., Bowman, P. J., Chamberlain, A. C., Verbyla, K., \& Goddard, M. E. (2009). Accuracy of genomic breeding values in multi-breed dairy cattle populations. Genetics Selection Evolution, 41, 51. doi 10.1186/1297-9686-41-51

Henderson, C. R. (1976). A simple method for computing the inverse of a numerator relationship matrix used in prediction of breeding values. Biometrics, 32, 69-83. doi 10.2307/2529339

Howard, J. T., Tiezzi, F., Pryce, J. E., \& Maltecca, C. (2017). Geno-Diver: A combined coalescence and forward-in-time simulator for populations undergoing selection for complex traits. Journal of Animal Breeding and Genetics, 134(6), 553-563. doi 10.1111/jbg.12277

Knol, E. F., Nielsen, B., \& Knap, P. W. (2016). Genomic selection in commercial pig breeding. Animal Front, 6(1), 15-22. doi 10.2527/af.2016-0003 
Koivula, M., Strandén, I., Pösö, J., Aamand, G. P., \& Mäntysaari, E. A. (2015). Single-step genomic evaluation using multitrait random regression model and test-day data. Journal of Dairy Science, 98 (4), 2775-2784. doi 10.3168/ jds.2014-8975

Lidauer, M., Strandén, I., Mäntysaari, E. A., Pösö, J., \& Kettunen, A. (1999). Solving large test-day models by iteration on data and preconditioned conjugate gradient. Journal of Dairy Science, 82, 2788-2796. doi 10.3168/jds. S0022-0302(99)75536-0

Long, N., Gianola, D., Rosa, G. J. M., \& Weigel, K. A. (2011). Long-term impacts of genome-enabled selection. Journal of Applied Genetics, 52, 467-480. doi 10.1007/s13353-011-0053-1

Lourenco, D. A. L., Misztal, I., Tsuruta, S., Aguilar, I., Lawlor, T. J., Forni, S., \& Weller, J. I. (2014). Are evaluations on young genotyped animals benefiting from the past generations? Journal of Dairy Science, 97(6), 3930-3942.

Lush, J. L. (1945). Animal breeding plans (3rd ed.). Ames, IA: lowa State University Press.

Meuwissen, T. H. E., Hayes, B., \& Goddard, M. E. (2001). Prediction of total genetic value using genome-wide dense marker maps. Genetics, 157(4), 1819-1829.

Meuwissen, T. H. E., \& Luo, Z. (1992). Computing inbreeding coefficients in large populations. Genetics Selection Evolution, 24, 305-313. doi 10.1186/1297-9686-24-4-305

Misztal, I., Legarra, A., \& Aguilar, I. (2014). Using recursion to compute the inverse of the genomic relationship matrix. Journal of Dairy Science, 97(6), 3943-3952. doi 10.3168/jds.2013-7752

Misztal, I., Tsuruta, S., Strabel, T., Auvray, B., Druet, T., \& Lee, D. H. (2002). BLUPF90 and related programs (BGF90). In: Proceedings of the 7th World Congress on Genetics Applied to Livestock Production, 19-23 Aug 2002, Montpellier. CDROM communication no. 28-07.

Sargolzaei, M., Chesnais, J. P., \& Schenkel, F. S. (2014). A new approach for efficient genotype imputation using information from relatives. BMC Genomics, 15, 478. doi 10.1186/1471-2164-15-478

VanRaden, P. M. (2008). Efficient methods to compute genomic predictions. Journal of Dairy Science, 91, 4414-4423. doi 10.3168/jds.2007-0980

Vitezica, Z. G., Aguilar, I., Misztal, I., \& Legarra, A. (2011). Bias in genomic predictions for populations under selection. Genetics Research, 93, 357-366. doi 10.1017/S001667231100022X

Weng, Z., Wolc, A., Shen, X., Fernando, R. L., Dekkers, J. C. M., Arango, J., . . . Garrick, D. J. (2016). Effects of number of training generations on genomic prediction for various traits in a layer chicken population. Genetics Selection Evolution, 48, 22. doi 10.1186/s12711-016-0198-9 
Table S1. The correlation ( $\pm 95 \%$ Confidence Interval) between the diagonal and off-diagonal values of $\mathbf{A}_{\mathbf{2 2}}{ }^{1}$ and $\mathbf{G}^{1}$ averaged across generations for both genotyping scenarios ${ }^{2}$

\begin{tabular}{|c|c|c|c|c|}
\hline \multirow{3}{*}{$\begin{array}{c}\text { Truncation } \\
\text { Point }\end{array}$} & \multicolumn{4}{|c|}{ Correlation Between A22 and G } \\
\cline { 2 - 5 } & \multicolumn{2}{|c|}{ Scenario1 } & \multicolumn{2}{c|}{ Scenario2 } \\
\cline { 2 - 5 } & $\begin{array}{c}\text { Diagonal } \\
\text { Elements }\end{array}$ & $\begin{array}{c}\text { Off-Diagonal } \\
\text { Elements }\end{array}$ & $\begin{array}{c}\text { Diagonal } \\
\text { Elements }\end{array}$ & $\begin{array}{c}\text { Off-Diagonal } \\
\text { Elements }\end{array}$ \\
\hline Full & $0.184(0.16-0.20)$ & $0.560(0.55-0.57)$ & $0.180(0.16-0.20)$ & $0.507(0.50-0.52)$ \\
\hline 10 & $0.165(0.14-0.18)$ & $0.566(0.56-0.58)$ & $0.188(0.17-0.21)$ & $0.513(0.50-0.52)$ \\
\hline 5 & $0.167(0.15-0.19)$ & $0.562(0.55-0.57)$ & $0.193(0.17-0.21)$ & $0.510(0.50-0.52)$ \\
\hline 4 & $0.164(0.14-0.18)$ & $0.559(0.55-0.57)$ & $0.192(0.17-0.21)$ & $0.506(0.50-0.52)$ \\
\hline 3 & $0.141(0.12-0.16)$ & $0.552(0.54-0.66)$ & $0.206(0.19-0.23)$ & $0.514(0.50-0.52)$ \\
\hline 2 & $0.200(0.18-0.22)$ & $0.577(0.57-0.59)$ & $0.223(0.20-0.24)$ & $0.517(0.51-0.53)$ \\
\hline 1 & $0.149(0.13-0.17)$ & $0.520(0.51-0.53)$ & $0.207(0.19-0.23)$ & $0.498(0.49-0.51)$ \\
\hline
\end{tabular}

${ }^{1} \mathbf{A}_{\mathbf{2 2}}$ matrix refers to the pedigree-based relationship for genotyped animals and $\mathbf{G}$ refers to the adjusted and weighted genomic relationship matrix used when generating the combined pedigree and genomic relationship matrix $(\mathbf{H})$.

${ }^{2}$ Scenario1: selection candidates did not have phenotype and genotype information when estimated breeding values were predicted. Scenario2: selection candidates had phenotype and genotype information when estimated breeding values were predicted.

${ }^{3}$ Truncation point refers to utilizing information on relatives and their associated progeny that are 1, 2, 3, 4, 5, 10 ancestral generation generations back from the selection candidates when breeding values were estimated. Under the Full truncation scenario all available information was utilized when EBV were estimated within each generation. 